\title{
Search for residual prostate cancer on pT0 radical prostatectomy after positive biopsy
}

\author{
Roberta Mazzucchelli • Francesca Barbisan • \\ Adriano Tagliabracci • Antonio Lopez-Beltran • \\ Liang Cheng • Marina Scarpelli • Rodolfo Montironi
}

Received: 6 November 2006 /Revised: 17 December 2006 / Accepted: 1 January 2007 /Published online: 7 February 2007

(C) Springer-Verlag 2007

\begin{abstract}
Reported incidence of no residual prostate cancer (i.e. pathological stage pT0) on radical prostatectomy ranges from 0.07 to $4.2 \%$. The incidence is higher after neoadjuvant endocrine treatment. The aim of this study was to search for residual cancer on radical prostatectomy (RP) specimens when an initial sampling failed to find the cancer in patients with positive biopsy. Our database of 1,328 consecutive patients whose biopsies and RP specimen were both examined at the Polytechnic University-United Hospitals of the Marche Region between March 1995 and June 2006 was reviewed. The radical prostatectomies were grossly completely sampled and examined with the whole mount technique. We identified eight patients (i.e. $0.6 \%$; three untreated and five hormonally treated preoperatively, i.e. 0.3 and $0.8 \%$, respectively, of the total number of RPs included in the study) with positive biopsy and with no
\end{abstract}

R. Mazzucchelli $\cdot$ F. Barbisan · M. Scarpelli $\cdot$ R. Montironi $(\bowtie)$ Section of Pathological Anatomy and Histopathology,

School of Medicine, United Hospitals,

Polytechnic University of the Marche Region (Ancona),

I-60020 Torrette,

Ancona, Italy

e-mail: r.montironi@univpm.it

A. Tagliabracci

Legal Medicine, School of Medicine,

Polytechnic University of the Marche Region (Ancona),

Ancona, Italy

A. Lopez-Beltran

Unit of Anatomic Pathology, Cordoba University Medical School,

Cordoba, Spain

L. Cheng

Department of Pathology and Laboratory Medicine,

Indiana University School of Medicine,

Indianapolis, IN, USA residual cancer in the initial routine histological examination of the RP. The RP of this group of eight was subjected to additional sectioning and evaluation of the paraffin blocks of the prostatectomy, also after block-flipping, immunostaining with an antibody against CAM 5.2, p63, PSA, and alpha-methylacyl-CoA racemase, and DNA specimen identity analysis. There were no cases with a false positive biopsy diagnosis, and cancer was not overlooked or missed in the initial routine histological examination of any of the 8 pT0 RPs. A minute focus of cancer (the diameter was always below $2.0 \mathrm{~mm}$ ) was found on the additional sections in five. In particular, cancer was found after block-flipping in one of them. In an additional case, cancer was eventually discovered after immunostaining tissue sections for cytokeratin CAM 5.2, for p63 and PSA. In the remaining two cases (one untreated and the other hormonally treated), cancer was not found $(0.15 \%$ of the 1,328 RPs included in the study); the review of the description of the macroscopic appearance of the RP and of its slides revealed that part of the peripheral zone corresponding to the site of the positive biopsy was missing, i.e. not removed from the patient at the time of the operation at least in one of the two. DNA specimen analysis confirmed the identity of the biopsy and prostatectomy in both. An extensive search for residual cancer reduces the number of pT0 RPs after a positive biopsy from 0.6 to $0.15 \%$. It is recommended to have the needle biopsy reviewed, carefully look again at the radical prostatectomy, do deeper sections and then flip certain paraffin blocks. In addition, atypical foci should be stained for basal cell markers and often AMACR, especially in hormone-treated cases. If a block is missing part of the peripheral zone (capsular incision), this should be commented on. DNA analysis for tissue identity should be performed when the other steps have been taken without finding cancer. 
Keywords Prostate cancer - Residual prostate cancer . Vanishing cancer

\section{Introduction}

In 1995, Goldstein et al. [9] introduced the term vanishing cancer phenomenon referring to cases with minute or no cancer on radical prostatectomy after a positive biopsy. They reported 13 patients with minimal (11 patients) or no (two patients) cancer in prostatectomy specimens. In two cases with no residual cancer on RP, they confirmed the identity of the biopsy and the prostatectomy tissue by DNA matching.

The Johns Hopkins Hospital group documented a fivefold increase in the incidence of no residual cancer on prostatectomy in patients in whom both biopsies and prostatectomies were performed at that institution between 1997 and $2005(0.07 \%$ in $1997,0.13 \%$ in 2004 and $0.34 \%$ in 2005) $[5,7,30]$. In their first study, they also documented two cases, one in which the biopsy review revealed only high-grade PIN and in which a diagnosis of cancer had been established in another institution, and a second case in which the possibility of specimen switching could not be ruled out due to DNA mismatch between the biopsy and the prostatectomy. Most recently, they have reported a series of 46 patients, 11 with no residual cancer on prostatectomy and 35 with minute cancer, and in 40 cases, they documented specimen identity [5]. In five of the six remaining cases, the results could not be interpreted due to technical problems, and in one case, the tissue from the biopsy with cancer did not match the tissue from the radical prostatectomy.

Bostwick and Bostwick [4] found that 38 patients with no cancer on prostatectomy, identified among 6,843 radical prostatectomies performed at Mayo Clinic during a 30-year period, showed no disease recurrence or progression after a mean follow-up of 10 years. In their experience, the incidence of vanishing cancer declined tenfold, comparing prostatectomies performed before 1980 (2.1\% incidence) to a more recent time interval from 1993 to $1995(0.2 \%$ incidence). They have estimated the current incidence of vanishing cancer at 2 per 1,000 radical prostatectomies [4].

Recently, Trpkov et al. [28] found an incidence of no residual cancer on prostatectomy of $0.67 \%$ after ten-core positive biopsy. They concluded that in most cases, finding no residual cancer on prostatectomy after additional sectioning and evaluation may indicate minimal patient disease. Very recently Zynger et al. [32] reported that their frequency of finding no residual cancer in RP specimens has increased from $0 \%$ in 2002 to $4.2 \%$ in 2006. In Canada, Srigley [25] has estimated the incidence of no residual cancer after positive biopsy in his practice at less than $0.5 \%$.
In Germany, the incidence of $\mathrm{pT} 0$ in patients with prostate cancer without neoadjuvant treatment was analysed by Herkommer et al. [13]. Based on a database of 3,609 patients, no residual prostate cancer was found in $0.8 \%$ after RP. Most men in this pT0 series had previous TURP or open prostatectomy, which may have eliminated prostate cancer, whereas in two patients, cytology was used for primary diagnosis. Only in $0.3 \%$ of men with positive prostate needle biopsies was no residual tumour detected in the prostatectomy specimen [13]. The incidence of pT0 patients in a very recent French study was reported to be $0.5 \%$ [6].

No residual cancer may also result from preoperative endocrine therapy $[11,12,15,16,19,24,26]$. The incidence of pT0 after neoadjuvant endocrine therapy for prostate cancer is reported to be higher than in untreated patients [11]. Kollermann et al. [16] analysed a group of 174 men who underwent prolonged androgen deprivation therapy and observed a pT0 in 36 of 174 men (21\%).

Several questions are addressed to explain why cancer is not present in a small proportions of prostatectomies. Cancer is missed during the examination of the RP, all prostatectomy tissue is not sampled, and the cancer is overdiagnosed in the biopsy. Pathologists face additional challenges in eliminating the possibility of laboratory error resulting in specimen (blocks, slides) mislabelling or switching and excluding an information system error resulting in specimen mix-up [28].

The aim of this paper was to report the results of a search for residual cancer on RP after an initial stage pT0 evaluation in eight patients with positive biopsy.

\section{Materials and methods}

We reviewed our database of 1,328 consecutive patients whose biopsies and RP specimen were examined between March 1995 and June 2006 at the five Pathological Anatomy Services associated with the Polytechnic University-United Hospitals of the Marche Region, Ancona, Italy. Most of the biopsies and all the RPs of this series of 1,328 patients were reported by the same pathologist (RM). Seven hundred patients were untreated before the operation, whereas 628 had received neoadjuvant endocrine treatment for approximately 3 to 6 months.

The biopsies and RPs were routinely processed as follows. The core biopsies from each site were submitted in separate containers. In brief, during biopsy processing, no more than two biopsy cores were embedded in paraffin blocks, and all blocks were sectioned at $3 \mu \mathrm{m}$ and were stained with haematoxylin-eosin (HE). All cores were sectioned in two separate levels represented on separate slides, with each slide containing three additional sections [28]. 
All radical prostatectomy specimens (tissue was not harvested for research) were fixed en bloc in $10 \%$ buffered formalin for at least $24 \mathrm{~h}$ and were grossly completely sampled using a standard protocol [17, 18, 28]. In particular, each specimen was painted over the surface with India ink. The seminal vesicles were amputated at the prostate junction and were grossly completely sampled. The remainder of the specimen was serially cut with a domestic electric food slicer calibrated and set to deliver slices which are $3-4 \mathrm{~mm}$ in thickness, representing transverse planes parallel to the initial apical and basal sections. Each prostate slide was processed into complete whole mount section, and one HE slide was routinely sectioned per block. The apical and basal transverse 2- to 3-mm margins were sectioned perpendicularly to assess the prostatic apical and basal margins. Prostatectomies were grossly sectioned in 8.5 slices (mean; range, 6-16), and 15.3 slides (mean; range, 10 to 20) were generated per prostatectomy. Slides from pelvic lymph nodes were not included in the reported number of examined slides.

We identified eight patients with positive 6- to 12-core biopsy and with no residual cancer (pathological stage pT0) in the initial routine histological examination of the RP and successively subjected to additional sectioning and evaluation.

\section{Search for residual cancer}

Since 1995, a special cancer searching protocol has been applied to those RPs in which an initial routine-based examination does not show residual cancer [18]. As mentioned earlier, this is basically done in those cases whose biopsies and RP specimen are both examined at the Ancona institution.

The following successive steps were undertaken by two pathologists (RM and $\mathrm{RMa}$ ). The procedure was usually stopped at the step where cancer is found.

1. The diagnostic needle biopsies were reviewed to exclude the possibility of a false positive biopsy diagnosis and to assess the approximate location of the biopsy with tumour, such as apex, mid-zone and base, both left and right.

2. The slides of the surgical specimens were reviewed for residual cancer that was initially overlooked or missed.

3. If the prostate was not totally embedded, the remaining prostate tissue was processed in toto. If the prostate was completely sampled, then this step was skipped. This type of information was usually contained in the pathology form where the all the steps of the processing procedure were recorded. The information was further confirmed by searching the specimen's container for residual pieces. As we routinely sample completely all prostatectomies, this step was skipped.

4. Additional deeper sections (i.e. three to five sections) of the prostatectomy area (paraffin block) corresponding to the location of the core with cancer were re-cut. Further sections were also obtained from all the other paraffin blocks.

5. Additional deeper sections (i.e. three to five sections) of the area corresponding to the location of the positive core as well as of all the remaining blocks were re-cut after block-flipping.

6. Immunostains for p63 and alpha-methylacyl-CoA racemase (AMACR) were performed to evaluate suspicious foci (When these two were not yet available, we used 34betaE12 immunostaining).

7. Immunostain for cytokeratin CAM 5.2, for p63 and for PSA (prostate specific antigen) was performed to identify the so-called "minimal residual cancer" especially in patients receiving neoadjuvant hormonal therapy [17].

8. Review the description of the macroscopic appearance of external and cut surfaces of the surgical specimen as well as inspect the contour of the tissue sections on the slides for hint or clues that might indicate that part of the tissue was missing either due to the surgical procedure or for technical reasons.

9. DNA specimen analysis was performed on formalinfixed tissue to confirm the identity of the biopsies and prostatectomies whenever necessary.

Table 1 Patients' clinical data

\begin{tabular}{llllll}
\hline Patient no. & Age (years) & PSA Prebiopsy $(\mathrm{ng} / \mathrm{ml})$ & Digital rectal examination & TRUS & Gland volume (cc) \\
\hline 1 & 68 & 9.0 & Abnormal & Normal & 47.9 \\
2 & 71 & 5.4 & Normal & Abnormal & 31.4 \\
3 & 64 & 6.1 & Normal & Normal & 28.1 \\
4 & 67 & 1.25 & Abnormal & Normal & 93.5 \\
5 & 60 & 5.9 & Normal & Normal & 36.6 \\
6 & 66 & 3.0 & Abnormal & Normal & 47.9 \\
7 & 58 & 3.9 & Normal & Abnormal & 29.3 \\
8 & 66 & 6.5 & Normal & Normal & 36.6 \\
Mean (range) & $65(58-71)$ & $5.1(1.25-9.0)$ & & $43.9(28.1-93.5)$ \\
\hline
\end{tabular}

PSA Prostate-specific antigen; TRUS transrectal ultrasound 
Table 2 Biopsy findings

\begin{tabular}{lllll}
\hline $\begin{array}{l}\text { Patient } \\
\text { no. }\end{array}$ & $\begin{array}{l}\text { No. of } \\
\text { positive } \\
\text { cores }\end{array}$ & $\begin{array}{l}\text { Gleason } \\
\text { score }\end{array}$ & $\begin{array}{l}\text { Cancer length } \\
\text { (\% of involvement) }\end{array}$ & $\begin{array}{l}\text { Positive core } \\
\text { location }\end{array}$ \\
\hline 1 & $1 / 12$ & $3+3=6$ & 5 & Right apex \\
2 & $1 / 10$ & $3+4=7$ & 10 & Right apex \\
3 & $1 / 6$ & $3+3=6$ & 5 & Left mid-zone \\
4 & $1 / 6$ & $3+3=6$ & 5 & Right mid-zone \\
5 & $1 / 6$ & $3+3=6$ & 5 & Right mid-zone \\
6 & $1 / 12$ & $3+3=6$ & 10 & Left mid-zone \\
7 & $1 / 10$ & $3+3=6$ & 5 & Right mid-zone \\
8 & $1 / 12$ & $3+3=6$ & 5 & Left base \\
\hline
\end{tabular}

DNA specimen identity analysis

Tested samples in an individual case included the biopsy core with cancer and a random block for the corresponding RP. The tissue was obtained as a direct section from formalin-fixed and paraffin-embedded blocks. DNA was extracted from paraffin-embedded tissue using the QIAmp DNA mini kit (Qiagen) according to manufacturer's protocol (Promega, Madison, WI, USA). Fifteen microsatellites and amelogenin locus were co-amplified by the AmpFlSTR identifiler kit (Applied Biosystems, Foster City, $\mathrm{CA}$ ), and the amplified fragments were electrophorized on an ABIPrism3130 genetic analyzer (Applied Biosystems). Fragment sizing and allele designation were established by GeneMapperID v3.2 software (Applied Biosystems), and genetic profiles from both biopsy and prostatectomy samples were compared.

\section{Results}

Residual cancer was not found in eight RPs after an initial routine examination. They represent $0.6 \%$ of the 1,328 consecutive patients. Three $(0.2 \%)$ of them were from the group of untreated patients, whereas five $(0.4 \%)$ were from those who had received neoadjuvant treatment. The latter figure represents $0.8 \%$ of the treated patients.
Fig. 2 Paraffin blocks, original sections and additional sections before and after block-flipping in case no. 1. a Is the right apex. b Is the left apex. c Includes the whole mount sections of the body of the prostate. d Is the right and left base. e Includes the seminal vesicles and deferens. A1 is the paraffin block. A2 is the original H-E-stained section. A3 includes the additional sections before block-flipping. A4 refers to the additional sections after block-flipping (Block-flipping was done only for $\mathbf{a}$ and $\mathbf{b}$ ). The dotted area (see also the red arrow) on the A4 slides is that of the cancer. It corresponds to the lesion of the biopsy seen in Fig. 1. The same identification procedure applies to $\mathbf{b}$. Concerning $\mathbf{c}$ and $\mathbf{d}$ the paraffin blocks, the original sections and the additional sections (block-flipping was not done) are shown. For e the paraffin blocks and the original sections are shown. (Other slides contain some annotations and abbreviations to indicate additional findings, slide orientation, and section order, etc.)

Patients' clinical data are summarised in Table 1. The mean age of the patients was 65 years (range, 58-71 years). Mean serum PSA pre-biopsy was $5.1 \mathrm{ng} / \mathrm{ml}$ (range, 1.25 $9.0 \mathrm{ng} / \mathrm{ml}$ ). Mean gland volume was $43.9 \mathrm{ml}$ (range, 28.1$93.5 \mathrm{ml}$ ). Digital rectal examination was abnormal in $37 \%$ of patients, and $25 \%$ of patients had abnormal transrectal ultrasound.

Patients' biopsy findings are shown in Table 2. In all patients, carcinoma was found in one core. Seven patients demonstrated biopsy Gleason score of $6(3+3)$, whereas in one, it was $7(3+4)$. Cancer occupied approximately $5 \%$ of the core length (Fig. 1) in 6 and $10 \%$ of the core in two. The positive biopsy location was variable, and there was no side predilection.

\section{Search for residual cancer}

There were no cases with a false positive biopsy diagnosis (step 1). Cancer was not overlooked or missed in any of the eight prostatectomies (step 2). Each prostate had been totally embedded (step 3 ).

Cancer was found on deeper sections (steps 4 and 5) in five cases. Cancer was in the right and left apex, respectively, in two of them. Cancer was in the mid-zone in another two, one in the left and the other in the right. The fifth was in the left base. In particular, the cancer found in

Fig. 1 Biopsy finding of a small groups of atypical acini (a) devoid of basal cells (b). Section immunostained for $\mathrm{p} 63$ (case no. 5). The diagnosis is acinar adenocarcinoma, Gleason score $3+3=6$. (The prostatectomy findings are those seen in Fig. 2)
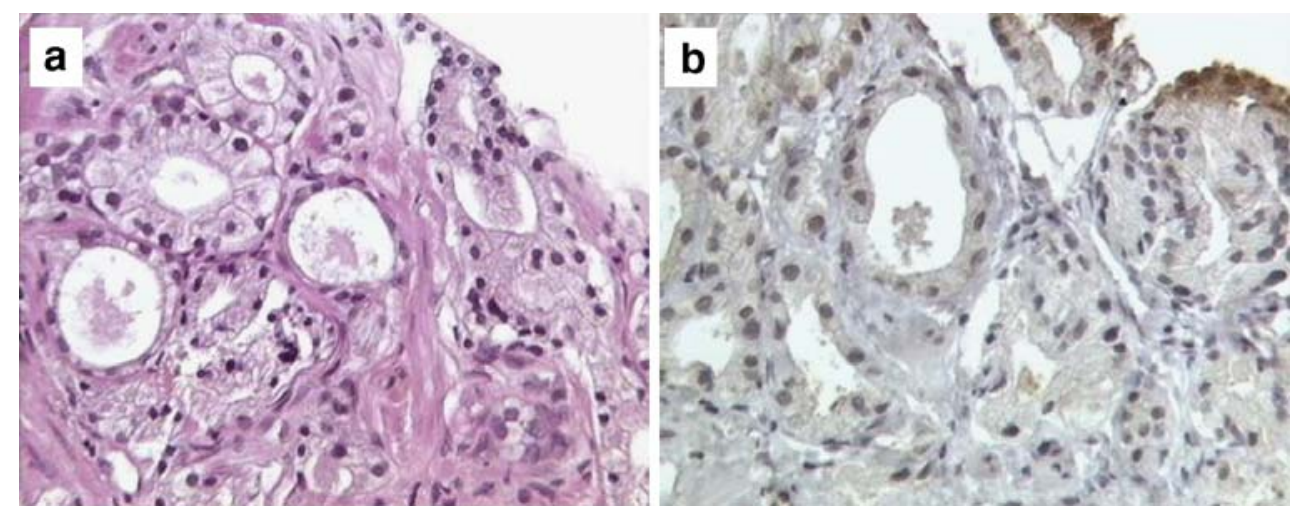


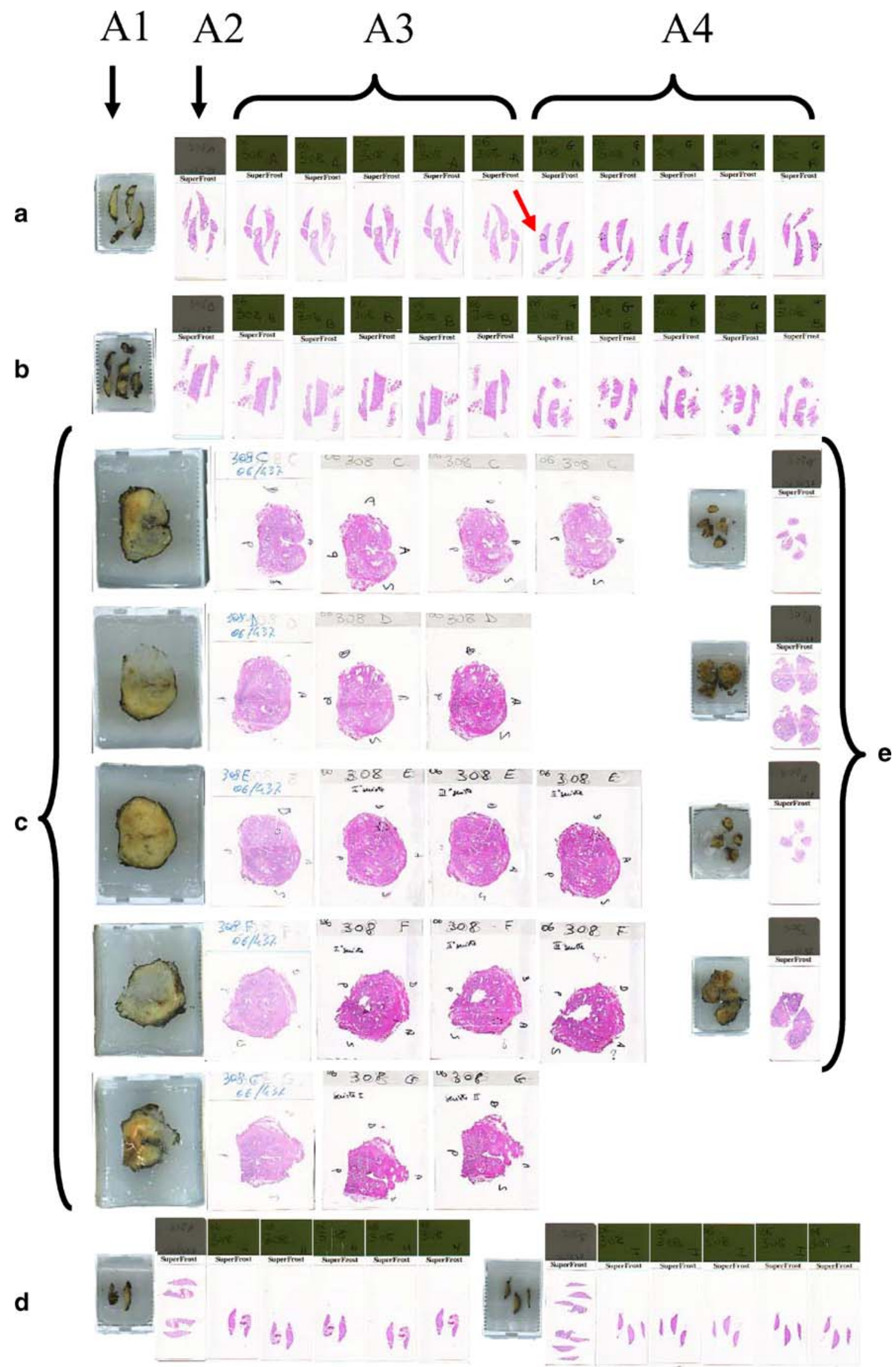


the right apex was discovered after block-flipping (i.e. step 5; Fig. 2). The location corresponded to site of the positive biopsy in four cases. Two out of five cases were from the untreated group and showed a Gleason score of $3+3=6$. Three cases had the morphologic appearance of cancer with evident regressive changes due to endocrine therapy. The diameter of the tumour, measured on the slides, was always below $2.0 \mathrm{~mm}$. In one of the three treated cases, an additional focus of cancer was seen at a distance from to that of the positive biopsy.

The definitive diagnosis was established on steps 6 and 7 in an additional case (the patient was hormonally treated before RP) where cancer was not seen in the additional sections even after block-flipping. Immunohistochemistry for p63 and AMACR was applied to a small suspicious focus of crowded acini. There were a few scattered p63 positive (basal) cells, whereas AMACR was negative. The focus was considered to be benign (i.e. atrophy). Cancer was discovered after immunostaining for cytokeratin CAM 5.2, for p63 and PSA. It was represented by scattered isolated cells positively immunostained with the antibody against CAM 5.2 (Fig. 3) and with PSA and negative for p63 (Fig. 3). The diameter of the focus was $1.0 \mathrm{~mm}$. The block selected for immunohistochemistry corresponded to an H\&E-stained slide with hypercellular stroma. There was no exact correspondence with the site of the positive biopsy.

In the remaining two cases (one untreated and the other treated preoperatively), cancer was not found on steps 4 through 7. The review of the description of the macroscopic appearance and the inspection of the contour of the tissue sections on the slides revealed that part of the peripheral zone of the prostate was missing (Fig. 4). This corresponded to the location of the positive biopsy (step 8). DNA specimen analysis confirmed the identity of the biopsy and prostatectomy in both cases (step 9; Table 3).

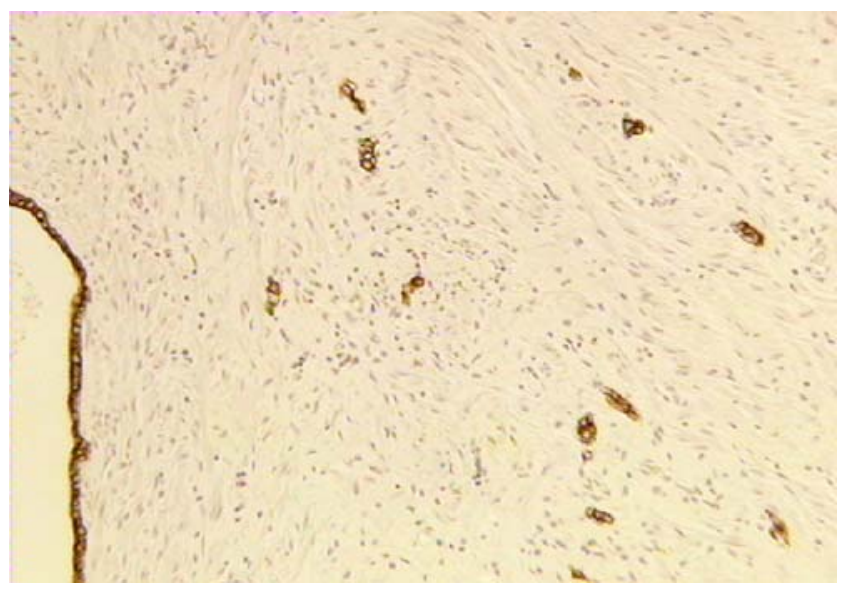

Fig. 3 Cancer is discovered after immunostaining for cytokeratin CAM 5.2 (case no. 6). It is represented by scattered isolated cells. The same cells are negative for the basal cell marker p63 and positive for PSA. Part of an atrophic duct is also present

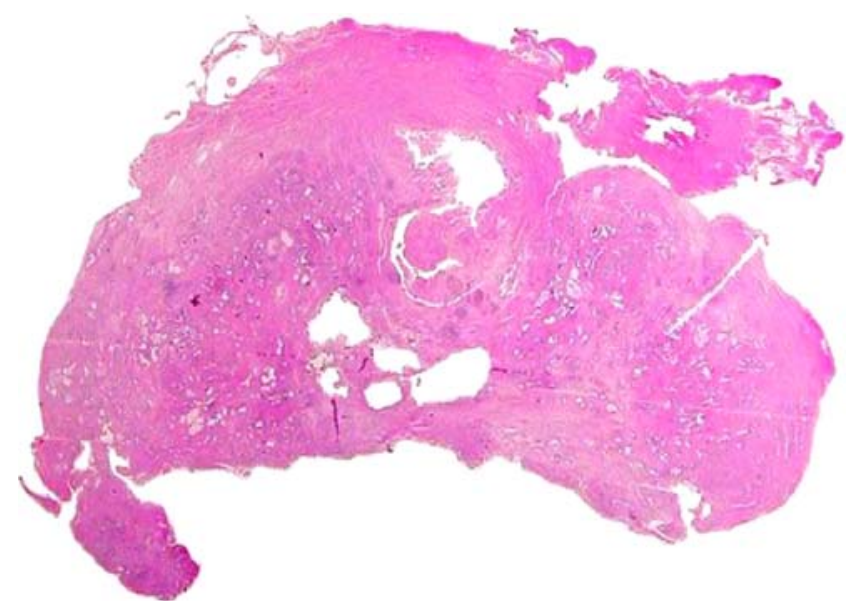

Fig. 4 Whole mount section (case no 7). Part of the peripheral zone, posteriorly, is missing

Incidentally, prostate tissue was documented clinically in one of the two patients (patient no. 7, hormonally treated before operation; the same patient whose prostate is shown in Fig. 4). In particular, a biopsy of the residual fragment showed normal prostate tissue and adenocarcinoma with features identical to those seen in the preoperative biopsy.

\section{Discussion}

Reported incidence of no residual cancer on RPs ranges from 0.07 to $4.2 \%(1-11,20)$. It is higher after neoadjuvant hormonal treatment [16].

There are multiple reasons why cancer may not be found on RP after a positive biopsy. In some cases, the cancer may be minute and completely removed by the initial procedure, either needle biopsy or transurethral resection of prostate. Small cancers may be removed from the RP during the technical preparation of the specimen, such as leveling of the paraffin blocks. Small cancer foci can also

Table 3 Results of the search for residual cancer

\begin{tabular}{llll}
\hline Patient no. & $\begin{array}{l}\text { Neoadjuvant } \\
\text { treatment }\end{array}$ & Cancer found & ID test done \\
\hline 1 & No & In recut & No \\
2 & No & In recut & No \\
3 & Yes & In recut & No \\
4 & Yes & In recut & No \\
5 & Yes & In recut after & No \\
& & block-flipping & \\
6 & Yes & After cytokeratin & No \\
7 & & stain & \\
8 & Yes & Not in RP & Yes, identical \\
\hline
\end{tabular}

${ }^{\mathrm{a}}$ A post-operative biopsy of the residual prostate fragment in the patient showed normal prostate tissue and adenocarcinoma with features identical to those seen in the preoperative biopsy. 
be completely obscured if the patient has undergone antiandrogen therapy or the RP shows extensive inflammation or granulomatous inflammation. Other reasons include false-positive diagnosis on the initial biopsy, resulting in cancer overdiagnosis, or false-negative diagnosis rendered on the RP. Review of the material by a second pathologist or a specialist in urological pathology can often resolve the latter scenario. Lastly, errors may occur either before a specimen is submitted for pathological assessment or in the pathology laboratory. These errors include switching of patients' requisitions, mislabelling or switching of specimen containers, mislabelling or switching slides or blocks and information system errors (e.g. incorrect case entry in the information system or mixed accession numbers) [28].

In our study, the incidence of RPs with no residual cancer after an initial routine examination of the 1,328 specimens was $0.6 \%$. This figure includes both untreated and hormonally treated patients. The incidence was 0.2 and $0.4 \%$, respectively, of the total number of RPs included in the study, or $0.4 \%$ (3 out 700 untreated patients) and $0.8 \%$ (5 out 628 treated patients) when the two groups were considered separately. The former is well in the range of values reported in the literature for untreated patients and very close to the figure published by Herkommer et al. [11, 13]. The latter value seen in our treated patients is much lower than that observed by Kollermann et al. [16].

Additional sectioning and evaluation of the cases can reduce the number of pT0 RPs after a positive biopsy. In particular, the current study showed that the final incidence was $0.15 \%$ and included only two RPs with missing parts, probably due to incomplete removal of the prostate. One of these two patients was hormonally treated preoperatively.

Our findings on the role of immunohistochemistry to detect residual $\mathrm{PCa}$ cells are in agreement with previous studies. Gleave et al. [8] found that $50 \%$ of the cases that exhibited no residual cancer on routine pathologic assessment had remaining foci of cancer discovered by immunostaining. Without the aid of additional step sections and immunostaining for cytokeratin, these cases would have been reported as being stage pT0 [2].

DNA identity on formalin-fixed tissue from the paraffin blocks is a useful test to establish specimen identity and to exclude the possibility of laboratory error when no residual cancer is found on RP after a positive biopsy. In particular, DNA analysis is a useful test that eliminates the possibility of specimen mishandling or switching by establishing the identity of the tissue from the positive biopsy and the RP. It can be performed in the formalin-fixed tissue from the paraffin blocks. Different methods are used to investigate tissue specimen identity. These include immunolabeling of blood group antigens [22], sex chromosome targeting using fluorescence in situ hybridization [21] and microsatellite analysis. According to some authors, microsatellite analysis is the gold standard for investigating tissue identity [1, 10 , $23,28,31]$.

In our study, the clinical and the biopsy data in patients with no initial residual cancer on radical prostatectomy after positive biopsy are similar to the findings from a recent study in patients with single-core positive biopsies and minimal cancer on biopsy $[28,29]$. The majority of patients with no residual cancer on prostatectomy demonstrated minute cancer foci in one or two biopsy cores with Gleason score of 6 , which, in many cases, may reflect minimal disease [28]. Rare small-volume cancers of higher grade may also be encountered [28]. Follow-up investigations have, in general, shown that no pT0 patient has clinical or biologic evidence of prostate cancer recurrence or progression $[6,14$, 20, 27].

There are four aspects that we have not explored in our study. One is that the carcinoma could be lost in facing off of the paraffin blocks. We are fully aware of this potential problem. Our technicians are instructed so that they have to collect tissue sections and not waste material when the paraffin blocks are levelled off. The second is whether the time, effort and expense of the sampling described in this paper are warranted on a routine basis for all pT0 cases. We have not done any analysis in these respects due to the fact that the number of cases is very small and that time and expense do not represent an issue of concern in our institution. The third is what degree of sampling would be necessary to serially section through the entire prostate. Probably thousands of sections would be required, and we were prepared to cut as many sections as needed to find cancer. The fourth is whether there is an outcome difference when the initial pT0 carcinomas are detected after more thorough sampling vs pT0 cases without additional sampling. This was not addressed in our study because the basic aim was to avoid that a pT0 report is rendered to the clinician and to the patient, thus triggering a potential legal issue with all the problems related to it.

There are few observations in the literature on pT0 cases and preoperative diagnosis of cancer made in TURP specimens. The real incidence of no residual cancer after cancer detected by TURP material is not known and is reported to be seen in 6 to $39 \%$ of cases [9], presumably due to tumour ablation during the initial resection. However, studies of this phenomenon are limited by variations in the number of tissue sections submitted for histological evaluation and by the small number of cases [3].

\section{Conclusions and recommendations}

The current study showed that an extensive search for residual cancer reduces the number of pT0 RPs after a positive biopsy. To achieve this, it is recommended to have 
the needle biopsy reviewed, carefully look again at the radical prostatectomy, do deeper sections and then flip certain paraffin blocks. In addition, atypical foci should be stained for basal cell markers and often AMACR, especially in hormone-treated cases. If a block is missing part of the peripheral zone (capsular incision), this should be commented on. DNA analysis for tissue identity should be performed when the other steps have been taken without finding cancer.

\section{References}

1. Abeln EC, van Kemenade FD, van Krieken JH, Cornelisse CJ (1995) Rapid identification of mixed up bladder biopsy specimens using polymorphic microsatellite markers. Diagn Mol Pathol 4:286-291

2. Bazinet M, Zheng W, Begin LR, Aprikian AG, Karakiewicz PI, Elhilali MM (1997) Morphologic changes induced by neoadjuvant androgen ablation may result in underdetection of positive surgical margins and capsular involvement by prostatic adenocarcinoma. Urology 49:721-725

3. Blackwell KL, Bostwick DG, Myers RP, Zincke H, Oesterling JE (1994) Combining prostate specific antigen with cancer and gland volume to predict more reliably pathological stage: the influence of prostate specific antigen cancer density. J Urol 151:1565-1570

4. Bostwick DG, Bostwick KC (2004) 'Vanishing' prostate cancer in radical prostatectomy specimens: incidence and long-term follow-up in 38 cases. BJU Int 94:57-58

5. Cao D, Hafez M, Berg K, Murphy K, Epstein JI (2005) Little or no residual prostate cancer at radical prostatectomy: vanishing cancer or switched specimen? A microsatellite analysis of specimen identity. Am J Surg Pathol 29:467-473

6. Descazeaud A, Zerbib M, Flam T, Vieillefond A, Debre B, Peyromaure M (2006) Can pT0 stage of prostate cancer be predicted before radical prostatectomy? Eur Urol 50:1248-1253

7. DiGiuseppe JA, Sauvageot J, Epstein JI (1997) Increasing incidence of minimal residual cancer in radical prostatectomy specimens. Am J Surg Pathol 21:174-178

8. Gleave ME, Goldenberg SL, Jones EC, Bruchovsky N, Kinahan J, Sullivan LD (1996) Optimal duration of neoadjuvant androgen withdrawal therapy before radical prostatectomy in clinically confined prostate cancer. Semin Urol Oncol 14(Suppl 2):39-47

9. Goldstein NS, Bégin LR, Grody WW, Novak JM, Qian J, Bostwick DG (1995) Minimal or no cancer in radical prostatectomy specimens: report of 13 cases of the "vanishing cancer phenomenon". Am J Surg Pathol 19:1002-1009

10. Gras E, Matias-Guiu X, Catasus L, Arguelles R, Cardona D, Prat J (2000) Application of microsatellite PCR techniques in the identification of mixed up tissue specimens in surgical pathology. J Clin Pathol 53:238-240

11. Hammerer P (2004) pT0 after radical prostatectomy: overtreatment for insignificant prostate cancer? Eur Urol 45:35

12. Hellstrom M, Haggman M, Brandstedt S, de la Torre M, Pedersen K, Jarlsfeldt I, Wijkstrom H, Busch C (1993) Histopathological changes in androgen-deprived localized prostatic cancer. A study in total prostatectomy specimens. Eur Urol 24:461-465

13. Herkommer K, Kuefer R, Gschwend JE, Hautmann RE, Volkmer BG (2004) Pathological T0 prostate cancer without neoadjuvant therapy: clinical presentation and follow-up. Eur Urol 45:36-41

14. Kollermann J, Caprano J, Budde A, Weidenfeld H, Weidenfeld M, Hopfenmuller W, Helpap B (2003) Follow-up of nondetectable prostate carcinoma (pT0) after prolonged PSA-monitored neoadjuvant hormonal therapy followed by radical prostatectomy. Urology 62:476-480
15. Kollermann J, Feek U, Muller H, Kaulfuss U, Oehler U, Helpap B, Kollermann MW (2000) Nondetected tumor (pT0) after prolonged, neoadjuvant treatment of localized prostatic carcinoma. Eur Urol 38:714-720

16. Kollermann J, Hopfenmuller W, Caprano J, Weidenfeld H, Weidenfeld M, Helpap B (2004) Prognosis of stage pT0 after prolonged neoadjuvant endocrine therapy of prostate cancer: a matched-pair analysis. Eur Urol 45:42-45

17. Montironi R, Mazzucchelli R, van der Kwast T (2003) Morphological assessment of radical prostatectomy specimens. A protocol with clinical relevance. Virchows Arch 442:211-217

18. Montironi $\mathrm{R}$, van der Kwast $\mathrm{T}$, Boccon-Gibod L, Bono AV, Boccon-Gibod L (2003) Handling and pathology reporting of radical prostatectomy specimens. Eur Urol 44:626-636

19. Murphy WM, Soloway MS, Barrows GH (1991) Pathologic changes associated with androgen deprivation therapy for prostate cancer. Cancer 68:821-828

20. Prayer-Galetti T, Gardiman M, Sacco E, Fracalanza S, Betto G, Pinto F (2007) The finding of no tumor (pT0) in patients submitted to radical retropubic prostatectomy for clinically localized prostate cancer. Anal Quant Cytol Histol (in press)

21. Riopel MA, Yu IT, Hruban RH, Lazenby AJ, Griffin CA, Perlman EJ (1995) Ideas in pathology: whose tumor is this? FISHing for the answer. Mod Pathol 8:456-457

22. Ritter JH, Sutton TD, Wick MR (1994) Use of immunostains to $\mathrm{ABH}$ blood group antigens to resolve problems in identity of tissue specimens. Arch Pathol Lab Med 118:293-297

23. Shibata D (1993) Identification of mismatched fixed specimens with a commercially available kit based on the polymerase chain reaction. Am J Clin Pathol 100:666-670

24. Smith DM, Murphy WM (1994) Histologic changes in prostate carcinomas treated with leuprolide (luteinizing hormone-releasing hormone effect). Distinction from poor tumor differentiation. Cancer 73:1472-1477

25. Srigley JR (2003) No cancer in the radical prostatectomy specimen: an approach to a surgical pathologist's dilemma. In: Microtutorial: a systematic approach to prostate needle biopsies. Canadian Association of Pathologists Annual Meeting. Prince Edward Island, Charlottetown, Section V: 32-36 (July 6)

26. Tetu B, Srigley JR, Boivin JC, Dupont A, Monfette G, Pinault S, Labrie F (1991) Effect of combination endocrine therapy (LHRH agonist and flutamide) on normal prostate and prostatic adenocarcinoma. A histopathologic and immunohistochemical study. Am J Surg Pathol 15:111-120

27. Thwaini A, Anjum F, Kaluba J, Shergill IS, Lewi HJE (2004) 'Vanishing' prostate cancer in radical prostatectomy specimens: incidence and long-term follow-up in 38 cases [letter/comment]. BJU Int 94:1145-1146

28. Trpkov K, Gao Y, Hay R, Yimaz A (2006) No residual cancer on radical prostatectomy after positive 10-core biopsy: incidence, biopsy findings, and DNA specimen identity analysis. Arch Pathol Lab Med 130:811-816

29. Trpkov K, Tsui J, Mancevska J, Faris P, Yilmaz A (2005) Single core positive and minimal prostate cancer $(\mathrm{PCa})$ on needle biopsy: correlation with tumor volume and clinically insignificant cancer. Mod Pathol 18(Suppl 1):167A

30. Truskinovsky AM, Sanderson H, Epstein JI (2004) Characterization of minute adenocarcinomas of prostate at radical prostatectomy. Urology 64:733-737

31. Worsham MJ, Wolman SR, Zarbo RJ (2001) Molecular approaches to identification of tissue contamination in surgical pathology sections. J Mol Diagnostics 3:11-15

32. Zynger DL, Dimov ND, Yang XJ (2006) Trends in carcinoma volume in 1612 radical prostatectomies: increased evidence of minimal and no residual cancer. Mod Pathol 19(Suppl 3):90A 